\title{
Le Trouble Psychosomatique: Poids du Passe, Trace Traumatique en Attente de Subjectivation
}

\author{
Nathalie Dumet ${ }^{1}$ \\ Université de Lyon - Université Lumière-Lyon 2
}

\begin{abstract}
RESUME - Le présent article propose de mettre en travail l'hypothèse selon laquelle le trouble psychosomatique constitue la trace traumatique d'une expérience passée en attente de subjectivation. Les cas de trois patientes, présentant chacune des manifestations somatiques spécifiques (obésité chez Mme Hera, douleurs physiques pour Christiane, somatisation gynécologique chez Philippa), permettent en effet de mettre en évidence la manière dont l'histoire subjective et surtout un vécu traumatique inélaboré prennent corps à travers leurs maux. Le trouble psychosomatique apparaît à ce titre chez ces sujets comme la rémanence d'une expérience vécue et non enregistrée psychiquement, mais susceptible de symbolisation à la faveur d'un dispositif d'invitation à la parole.
\end{abstract}

Mots-clefs: agir; mémoire; subjectivation; symbolisation; trouble psychosomatique.

\section{A Perturbação Psicossomática: Peso da Passagem, Vestígio Traumático a Espera de Subjetivação}

\begin{abstract}
RESUMO - O presente artigo propõe discutir a hipótese de que a perturbação psicossomática constitui um vestígio traumático de uma experiência passada na espera de subjetivação. Os casos de três pacientes que apresentam manifestações somáticas específicas (Sra. Hera, obesidade; Christiane, dores físicas; Philippa, somatização ginecológica) permitem colocar em evidência a maneira como a história subjetiva e principalmente os estados traumáticos não-elaborados materializam-se através de seus males somáticos. As perturbações psicossomáticas aparecem nesses sujeitos como um vestígio remanescente de uma experiência vivida e não registrada psiquicamente, mas suscetível de simbolização por meio do dispositivo da linguagem verbal.
\end{abstract}

Palavras-chave: ação; memória; problema psicossomático; subjetivação; simbolização.

\section{The Psychosomatic Trouble: The Weight of the Cross, Traumatic Trace Looking Forward the Subjectivation}

\begin{abstract}
This text proposes to discuss the hypothesis according to psychosomatic trouble constitute a traumatic trace of a past experience looking forward to subjectivation. Three patients' cases with specific somatic manifestations (obesity for Mrs. Hera, aches for Christiane, genital somatization for Philippa) permit to show how subjective history and a traumatic state materialize with somatic troubles. The psychosomatic trouble appears as the trace of an experience psychically undiscribed. Symbolisation appears possible when there is a situation to favour verbal language.
\end{abstract}

Key words: acting; memory; psychosomatic trouble; subjectivation; symbolization.

\section{Préambule...}

Un extrait clinique servira d'entrée en matière.

Mme Hera ${ }^{2}$ est âgée de 40 ans quand nous la rencontrons en service de médecine où elle est venue consulter pour traiter

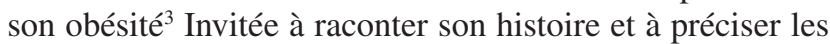
conditions de constitution de son obésité, Mme Hera explique que son poids est toujours resté stable entre 18 et 35 ans; elle

1 Endereço: Institut de Psychologie, Université Lumière-Lyon 2, 5 avenue P. Mendès, France 11-69676 BRON. E-mail: nathalie.dumet@univ-lyon2.fr

2 Ainsi nommée par allusion à la célèbre déesse grecque, épouse de Zeus, du même nom car comme celle-ci, cette patiente a connu trahison et infidélité conjugales.

3 Elle pèse alors 100 kilos pour une taille de $1,60 \mathrm{~m}$, soit un indice de masse corporelle de $39 \mathrm{~kg} / \mathrm{m}^{2}$. a certes grossi lors de chacune de ses trois maternités, mais ses kilos de grossesse ont toujours été rapidement reperdus dans l'après-coup de ses accouchements. Mme Hera précise encore qu'il y a cinq ans, elle a perdu 10 kilos en très peu de temps après que son conjoint l'ait quittée, pour aller vivre avec une autre femme plus jeune, la laissant alors seule avec leurs quatre enfants (dont deux, des jumeaux, en bas âge). A peine a-t-elle maigri qu'elle reprend tout le poids perdu et bien davantage puisque son obésité se constitue dans l'année qui suit cette séparation. Mme Hera relate alors son vécu de la manière suivante. "Sur le coup, je n'ai pas compris... comme ça, du jour au lendemain, il est parti". Après un temps de latence, elle ajoute: "j'ai l'impression d'avoir passé ces dernières années dans une grotte. Enfermée, recluse... J'ai survécu mais je ne sais pas comment j'ai fait... J'ai été trahie, 
abandonnée pour une autre. (latence) Il [son mari] est parti pour une autre (...). Il est parti et m'a laissé toute la charge des enfants, la charge de les éduquer, toute seule, sans personne (latence). Vous comprenez c'était un poids. Au début je me sentais pas la force d'y arriver. J'avais jamais travaillé... et pas d'argent... et encore deux bambins. C'était trop... trop lourd... (latence)... et puis un beau jour je me suis dit que je ne pouvais plus vivre comme ça, il fallait que je réagisse".

Ce court extrait clinique - tant la situation somatique de Mme Hera que ses paroles, et certaines plus que d'autres - introduit d'emblée notre propos consacré au poids du passé. Poids du passé à entendre certes ici au sens propre puisqu'il est bien sûr question chez Mme Hera de ses troubles du poids apparus quelques années en arrière à la faveur d'un événement désorganisateur (le départ de son mari vécu comme un abandon). Mais poids du passé à entendre également au sens figuré, car dans ce cas comme dans les deux autres qui suivront (Christiane et Philippa), il sera justement question de la pesanteur ou persistance de l'histoire passée se donnant à entendre sous des expressions symptomatiques somatiques variées et autres que strictement pondérales. Reste au passage que cette dimension littérale ou figurée (du trouble somatique) n'est en soi pas une mince affaire (!); elle engage justement toute une réflexion sur le rapport du sujet au corps, à la psyché, au langage et au registre symbolique - points sur lesquels nous aurons justement l'occasion de revenir.

Poids du passé, donc, qui fait plus précisément référence ici à la manière dont l'histoire subjective s'incarne, à la manière dont l'expérience passée et ses effets s'inscrivent chez l'individu, en l'occurrence prennent corps à travers le trouble dit psychosomatique ${ }^{4}$.

A côté des différents types de mémoire existants (conscient, inconscient,...), nous envisagerons ici l'idée selon laquelle le corps constitue une forme de mémoire à part entière ou plutôt une modalité de mémorisation. Plus précisément, l'hypothèse que nous proposons de mettre en travail dans ce présent article est la suivante: le trouble psychosomatique constitue la trace traumatique d'une expérience passée en attente de subjectivation.

\section{Rappel: quelques jalons théoriques pour penser les rapports existant entre corps et mémoire}

Cette thématique des rapports existant entre "Corps et mémoire" n'est nullement nouvelle. Il n'y a qu'à parcourir la littérature du 19ème siècle consacrée aux recherches menées dans les champs de la psychophysiologie, de la neurologie et même de la neuropathologie, recherches destinées à identifier la ou les structures organiques, cérébrales, sièges de la mémoire. Ces travaux plaident donc d'emblée pour notre propos mais dans un champ théorique connexe au nôtre. Qu'en est-il du côté psychanalytique?

Le lien corps-mémoire est une thématique dont on peut dire qu'elle a été coextensive de la naissance de la psycha-

4 Ou encore trouble somatique, car il n' existe selon nous, suivant en cela la conception de Célérier (1989) par exemple, aucune frontière entre trouble somatique et trouble psychosomatique. Il existe des troubles dont l'expression est manifestement de nature somatique (à la différences d'autres troubles dont l'expression est plus strictement psychique) mais dont l'étiologie est polyfactorielle. nalyse puisque Freud (1912/1953) a très vite compris les troubles névrotiques dans leur connexion avec des contenus psychiques oubliés, refoulés. "Le névrotique souffre de ses souvenirs", écrivait-il en effet en 1912 (in "La dynamique du transfert"). Freud a aussi montré comment les troubles de conversion, affectant le corps fonctionnel du sujet, apparaissaient comme le déplacement et la traduction au plan corporel - la conversion proprement dite - du conflit intra-psychique opposant chez le sujet désirs sexuels et interdits. Tous ces travaux freudiens ont de plus abouti à la formule bien connue selon laquelle "les hystériques souffrent de réminiscences". Nous reviendrons plus tard sur l'hystérique et son rapport à la mémoire, ne serait-ce que par opposition à une autre catégorie de manifestations, à savoir les troubles somatiques ou psychosomatiques qui engagent, eux, le corps réel et non pas seulement le corps imaginaire (comme c'est le cas dans la conversion). Mais pour l'instant poursuivons très succinctement les pistes de réflexion entamées par certains auteurs autour des rapports entre corps et mémoire.

Après les travaux freudiens donc, et bien que s'écartant peu ou prou du référentiel psychanalytique, on ne peut éviter de mentionner les travaux des fondateurs des thérapies psycho-corporelles. A la tête de ces mouvements, Reich, disciple et dissident de Freud, mais aussi ses "descendants", Lowen, Janov pour n'en citer que quelques uns. Bien que chacun de ces auteurs ait forgé sa propre explication et sa propre thérapeutique des souffrances humaines, leur communauté de pensée repose sur l'idée de la nécessaire mobilisation et expression corporelle, pour ne pas dire décharge physique, du patient, de manière à ce que celui-ci puisse se défaire de ses traumatismes - les évacuer. Le point commun de toutes ces approches réside au fond dans le postulat selon lequel le corps conserverait la trace, l'inscription des souffrances et traumatismes du sujet. Celles et ceux-ci siégeraient au plus profond de l'être, à savoir dans la chair même de l'individu. "Le corps se souvient", tel est d'ailleurs le titre même d'un ouvrage de Janov (1996). Ainsi, selon ces approches, le patient devrait-il revivre dans sa vie présente (et à l'occasion des séances psychocorporelles) les traumas anciens afin de s'abstraire de ces chaînes du passé ayant marqué son être sous la forme de blocages caractériels, corporels ou de “cuirasses musculaires" encore. Afin d'éliminer les tensions (physico-psychiques) demeurées inscrites en lui et abréagir ce qui n'a pu l'être en temps opportun, c'est-à-dire au temps jadis, il faudrait au sujet repasser par la voie inaugurale selon laquelle le traumatisme a été vécu, enregistré: en l'occurrence il s'agirait de revivre physiquement les traumatismes vécus (à commencer par celui originel de la naissance).

On connaît les limites de ces approches fondées quasi-essentiellement en leur temps sur des considérations économico-énergétiques et les critiques ne manquent pas à l'égard de ces thérapies comme des assertions théoriques les sous-tendant - à commencer par celle consistant à dire que le sujet revit sa naissance; revivre physiquement les conditions de sa naissance est impossible pour la simple raison que le sujet, adulte, est dans des dispositions, physiques et psychiques, autres que celles qui caractérisaient son être le jour de sa venue au monde. Lors de ces thérapies, le sujet ne peut donc qu'agir, mettre en acte et en scène une figuration ou 
représentation de ce qu'il pense être sa naissance ou le vécu de celle-ci mais pas celle-ci à proprement parler.

Toutefois, et malgré ces réserves et critiques, n'en demeure pas moins intéressante ici l'idée que le corps conserverait l'empreinte de ces expériences passées traumatiques. Le corps serait en somme le dépositaire de ce qui, de douloureux notamment, est arrivé à l'individu. Le corps et ses maux éventuels seraient comme la mémoire vive en quelque sorte du sujet, mémoire qu'il serait alors possible d'activer pour accéder aux souffrances enfouies et permettre leur liquidation, leur métabolisation.

Nous ne nous appesantirons pas davantage sur ces travaux déjà désuets mais qui ne pouvaient être totalement passés sous silence dans le présent champ de recherche. Pour rester sur des références non psychanalytiques, évoquons les travaux contemporains issus des recherches en neurosciences, cognitives notamment, où l'on s'intéresse justement à "l'inscription corporelle de l'esprit" (pour reprendre là encore le titre d'un ouvrage, celui de Varela, 1993). En lien, on ne peut également manquer de citer les recherches récentes de certains cliniciens (tel par exemple, Kandel, 2002), destinées à mettre en évidence les modifications biologiques suscitées par le travail psychique et psychothérapique; tous travaux soulignant donc, là encore, l'existence d'un engramme au plan biologique ou cellulaire des états et vécus subjectifs et même des changements intrapsychiques.

\section{L'obésité, un trouble psychosomatique, une souffrance en attente de symbolisation}

Laissons momentanément de côté cette revue théorique, non exhaustive, pour revenir au cas de Mme Hera. Que nous donne-t-il à percevoir?

Au moment où son histoire prend un nouveau cours, de par le départ de son conjoint, Mme Hera prend un nouveau corps: son fonctionnement somatique devient en l'espace de quelques mois gros, lourd, pesant, surchargé, ... autrement dit, il devient accablé des effets psychiques et affects mêmes que la patiente exprime le jour de la rencontre psychologique, cinq ans plus tard; affects et représentations que la patiente déclare - rétrospectivement il est important de le noter - avoir éprouvé. Mais de quel(s) temps ces affects sont-ils concomitants au juste? Du moment où est survenu l'abandon (de ce qui a été vécu comme tel)? De l'aprèscoup de celui-ci? Et/ou aussi du moment présent?

Ces mots - rappelons-les: 'toute la charge, c'était un poids, pas la force, c'était trop, ... trop lourd" - la patiente n'est vraisemblablement en mesure de les prononcer qu'en aprèscoup, c'est-à-dire cinq ans après le choc, lors de sa récente démarche de consultation. Celle-ci constitue un mouvement destiné à se dégager de l'enfermement dans lequel elle s'est jusqu'alors maintenue, à se dégager de l'obésité dans laquelle elle (s')est aussi enfermée depuis cette date. Il faut ici reconnaître que Mme Hera est encline à ce travail d'expression verbale de narration, de remémoration et de liaison entre hier et aujourd'hui, parce qu'un dispositif d'invitation à la parole (initié par un Autre) est mis à sa disposition - dispositif proche de ce que Del Volgo (1993) a décrit en clinique médicale sous le terme d" "instant de dire"5.

5 A la nuance suivante ici, à savoir qu'outre la traduction langagière de ce que le sujet somatique vit présentement, la patiente articule aussi présent et passé.
Pour en revenir à ce qui s'est passé chez Mme Hera, il semble bien que cinq ans plus tôt, trop désemparée, abasourdie, voire sidérée, sujette à de majeures angoisses d'abandon ${ }^{6}$ il semble bien que Mme Hera n'ait trouvé que son corps et ses maux (perte puis prise de poids) pour surseoir à sa douleur et même pour parer à un effondrement dépressivonarcissique certain; "pour survivre", aussi, comme elle l'a dit elle-même.

L'affect - dépressif, d'angoisse, de douleur - s'est-il ici converti dans le soma, à l'instar du destin de l'affect en jeu dans le procédé hystérique? Assurément non. D'abord il n'est point question des mêmes affects; la problématique génitale est bien loin d'être de mise chez Mme Hera, ses vécus et souffrances ressortissent davantage à une problématique narcissique-limite; de plus, son trouble somatique - l'obésité ici - n'est pas un simple trouble fonctionnel comme on peut souvent l'observer dans les symptômes de conversion?. L'obésité entame le soma de Mme Hera, tant du point de vue de sa réalité externe (l'enveloppe corporelle proprement dite) que du point de vue de sa réalité cellulaire interne: c'est en effet la biologie, l'ensemble des cycles métaboliques en jeu dans la régulation énergétique et par voie de conséquence dans la régulation pondérale qui se trouvent ici altérés et mis à mal chez la patiente. L'obésité, un trouble psychosomatique? Ou plutôt: le trouble pondéral de Mme Hera, la survenue de son obésité auraient-ils donc quelque chose à voir avec ce qui est habituellement en jeu dans les troubles dits psychosomatiques?

C'est ici que nous entrons plus avant dans le vif de notre propos et qu'il importe de préciser d'autres données théoriques, issues du corpus psychanalytique.

Les troubles somatiques (ou dits psychosomatiques) passent dans la littérature spécialisée depuis les travaux de Marty $(1976,1980,1990)$ et collaborateurs, pour résulter de difficultés psychiques sinon même de la défaite du psychisme chez le sujet. A défaut de pouvoir être traitées psychiquement, certaines situations ou excitations, en excès, seraient drainées puis déchargées vers la voie organique. En cela, ces troubles physiques ne suivraient pas le symbolisme corporel de l'hystérique puisqu'ils échapperaient par définition à une saisie mentale; de même ils ne sauraient être porteurs d'un quelconque conflit, ni même vecteurs d'un quelconque fantasme (selon le courant psychosomatique martyen en tous les cas).

Les troubles somatiques ou psychosomatiques constitueraient donc à cet égard un paradigme de ce qui, chez le sujet, échoue à être signifié, représenté, élaboré mentalement et verbalement. Ces troubles renverraient à des contenus en souffrance d'appropriation subjective. En souffrance, c'està-dire alors peut-être aussi en instance d'historicisation, en instance de subjectivation et donc de symbolisation - comme on va justement le voir à l'aide d'un second cas dans lequel le "poids du passé" se donne à percevoir sous une forme symptomatique toutefois moins littérale que chez Mme Hera avec son trouble pondéral.

6 Angoisses derrière lesquelles se profilait certainement le spectre de l'effondrement psychique et narcissique.

7 Même si l'on sait bien que la réalité médicale du trouble n'est pas un argument suffisant pour soutenir, ou au contraire éradiquer, la signification psychonévrotique de celui-ci. 


\section{Christiane, ou la douleur physique pour nier et faire exister à la fois le trauma}

Il s'agit du cas de Christiane ${ }^{8}$, jeune femme d'une vingtaine d'années venue nous consulter pour de nombreux troubles somatiques et un mal de vivre; sans parler de nombreuses douleurs dans son corps qui ne la lâchent pas - à moins qu'il ne s'agisse de l'inverse... En effet, la douleur physique semble être le pivot organisateur de la vie de Christiane, l'axe vertébral qui lui permet de tenir debout, aux sens propre et figuré du terme... La douleur est surtout pour elle, comme l'écrit L'Heureux-LeBeuf (2002, p. 16) à propos du malade hypocondriaque, "un compagnon 'fiable', le seul qui ne l'abandonne jamais".

Christiane, donc, à l'occasion d'une séance de psychothérapie, revient pour la énième fois sur son corps douloureux. Jusqu'à cette séance, toute tentative de la faire penser, associer autour de cette douleur persistante, de ce corps de douleurs, de cette présence douloureuse, la sienne..., s'était avérée vaine. Ce jour là en revanche, Christiane dit: "la douleur c'est ma mémoire, si je la perds, je n'existe pas". Je reprends interrogativement: "votre mémoire?" et Christiane poursuit en énumérant certains pans de son histoire déjà abordés ensemble lors de séances antérieures (tels que l'inconsistance de la figure paternelle, la maladie mentale de sa mère, de multiples séparations affectives au cours de la prime enfance, le climat d'instabilité dans lequel elle a vécu, etc.). Puis Christiane mentionne les attouchements sexuels dont elle a, petite fille, été l'objet de la part d'un ami de ses parents de passage chez eux. Elle avait rapidement fait allusion à cette scène d'attouchements une fois auparavant, à vrai dire le premier jour où je l'avais reçue, mêlant alors cet événement, très vague souvenir au demeurant, en vrac avec bien d'autres, sur la longue liste de ce qui, selon elle, la faisait souffrir (psychiquement...) et pour lequel elle souhaitait justement consulter. Jusqu'à ce jour elle n'avait plus reparlé de cette violation de son intimité - même si de mon côté le contenu de certaines séances et plus encore la tendance de Christiane à supporter répétitivement des rapports sexuels douloureux avec des hommes de passage n' avaient pas manqué de me rappeler cette fugace évocation du premier jour. Lors de la séance dont il est ici question, Christiane revient donc d'elle-même à cette scène infantile. A peine y a-t-elle fait allusion qu'elle fait volte-face, se bloque, ne veut, ne peut en dire davantage, elle est mal, elle a mal, elle frissonne. Peu à peu, avec mon soutien, elle parviendra à retrouver et nommer ce que jusqu' alors, de son histoire, et de cette histoire là en particulier, elle avait exclu d'elle. La suite de la séance et d'autres qui suivront vont en effet permettre à Christiane d'identifier et de s'approprier enfin des vécus jusqu'alors impensés, expulsés de sa psyché, et ce justement en vue de sauvegarder son intégrité psychique ${ }^{9}$.

Parler de cette scène infantile est douloureux pour Christiane à plus d'un titre. En parler "pour de vrai" comme elle

8 Ce cas a déjà été utilisé pour montrer les effets de la rencontre intersubjective sur le corps du clinicien; pour de plus amples développements, le lecteur pourra se reporter à Dumet (2004).

9 Un semblant d'intégrité psychique, faudrait-il plutôt dire, tant Christiane, radicalement coupée de certaines parties d'elle-même, de sa vie émotionnelle, coupée de pans de son histoire affective, vivait alors dans un état de chaos, de morcellement intérieur. dit, c'est raviver cette scène, et donc son vécu, proscrit; c'est activer celui-ci. C'est alors pour Christiane l'exposer à vivre (plus que revivre) les vécus et affects qu'elle a jusqu'alors voulu ignorer, oublier, plus encore forclore. Pourquoi? Parce qu'accepter la réalité de cette scène ne permet plus de faire comme si cela ne s'était pas produit; c'est accepter que ce soit vrai, que cela ait eu lieu "pour de vrai", réellement. Parler, c'est donc non seulement raviver la trace de cette scène et l'imprimer dans sa mémoire, dans sa topique, mais c'est aussi et surtout accepter la réalité de ce qui s'est passé, de ce que ses sens ont enregistré. Lors de cette scène, Christiane a ressenti ${ }^{10}$ un mélange de plaisir et d'horreur. Du "plaisir", car enfin quelqu'un, un adulte, qui plus est substitut paternel, s'est occupé d'elle; de l'horreur aussi, car elle n'a sur le moment pas compris ce qui se passait sinon vaguement. Lors de cette séance thérapeutique, Christiane pourra alors dire la chose suivante: les doigts de cet homme sur son corps et sur son sexe de fillette, elle sait maintenant, elle le ressent, elle se souvient, ça lui faisait mal alors.

Parler de cette situation, c'était donc, pour Christiane, (re)vivre ce plaisir teinté de douleurs, c'était surtout reconnaître la réalité de cette scène et par là même la réalité de l'abus. Ça s'était réellement passé; elle, Christiane, elle ne pouvait pas/ou ne pourrait plus faire l'économie de ce vécu (devenu dès lors souvenir - l'inscription temporelle venant ici de faire son apparition). Elle ne pouvait/pourrait plus fonctionner dorénavant comme si cela n'avait pas eu lieu, comme si ce n'était pas vrai...

Mais cette réalité n'était horrible pour Christiane que parce qu'elle en dissimulait une autre, aussi sinon plus douloureuse encore pour elle, celle là même contre laquelle elle s'évertuait à lutter depuis toujours (et depuis le début de la thérapie donc aussi). Reconnaître la réalité de cette scène de son existence exposait en effet Christiane à voir et à devoir reconnaître la carence parentale, l'insuffisante attention et protection parentale à l'égard de la jeune enfant qu'elle était. Et là encore, en mettant maintenant cette perception (ce vécu?) en représentation et en souvenir, voire aussi en dévoilant haut et fort cette réalité à un Autre, Christiane ne pouvait/ne pourrait plus nier cette réalité parentale ô combien décevante et douloureuse parce que fortement carencée. Il faut ici préciser que la psychogenèse de Christiane s'est en effet organisée autour d'échanges, et d'échanges corporels notamment, durs, sévères, froids, mais aussi de manques, laissant dès lors subsister des vécus chaotiques et un corps de douleurs, seule manière pour Christiane de s'arrimer dans l'existence et de trouver l'objet. En somme, c'est comme si Christiane disait: "mieux vaut un mauvais objet qui me fait vivre, même dans l'aliénation et la souffrance, plutôt qu'une absence complète d'objet qui me conduirait à la mort' (Stoloff, 2000, p. 78).

Outre ces aspects, l'expression somatique douloureuse chez Christiane a aussi, comme on vient de le voir, vocation d'oubli, tentative d'évacuer, de rejeter ce qui résiste à l'inscription psychique et subjective. Mais l'expression somatique tient encore et simultanément lieu de mémoire, de témoin et de zone d'inscription de vécus indicibles, irreprésentés, voire peut-être irreprésentables. Son corps se rappelle au "bon souvenir" de Christiane - si on ose cette expression

10 Tel est ce que nous parviendrons progressivement à (re)constituer ensemble. 
- sauf qu'ici il est surtout question de vécus douloureux et délétères, et d'une intensité telle que cela a empêché leur inscription psychique, leur mise en représentations et en souvenirs véritables; le corps, lui en revanche, a gardé trace - au sens propre du terme - de cette douleur, il s'est en quelque sorte fait porte-douleur.

\section{Le trouble psychosomatique: rémanence d'une expérience vécue mais non enregistrée psychiquement}

Ce que les cas de Mme Hera et de Christiane montrent et amènent à penser, c'est donc la manière dont le corps du sujet, via certaines expressions symptomatiques, peut garder la trace, la "souvenance" de certaines expériences, des expériences traumatiques assurément, et de leurs effets produits sur le sujet.

Certes, on pourrait être tenté de penser, en regard de la théorie freudienne ou martyenne du traumatisme, que c'est l'excès de tensions dans ces différentes situations de vie - qu'il s'agisse de moments de la psychogenèse pour Christiane ou d'une épreuve d'actualité pour Mme Hera - qui a abrasé l'appareil psychique de ces patientes; c'est le trauma qui a débordé leur capacité de pare-excitation, excédé leurs possibilités (respectives et différentielles) de liaison et d'élaboration mentale, désorganisé leur équilibre psychique et par voie de conséquence a abouti chez elles à une désorganisation somatique, entraînant la survenue d'un trouble psychosomatique (obésité pour Mme Hera, divers troubles somatiques et surtout des douleurs physiques chez Christiane). Mais s'en tenir à cette vision strictement économique du trauma et de son traitement (un non traitement mental) reviendrait à négliger un paramètre important, à savoir que, chez ces patientes, les troubles somatiques expriment et traduisent plus encore la rémanence des expériences vécues mais non enregistrées psychiquement (autrement dit non subjectivées).

La rémanence, cela désigne, en psychologie, "la propriété qu'ont certaines sensations ou images de laisser subsister un certain temps l'excitation qui leur a donné naissance" (Dictionnaire Larousse, 1993, p. 1605). La rémanence, c'est donc la persistance, la "perduration"11 de certains vécus, le maintien durable des effets produits sur le sujet par certaines expériences ou situations marquantes.

La rémanence, telle est bien ce qui caractérise, chacune à leur manière, Mme Hera et Christiane, ainsi que leur psychosoma:

- le corps de Mme Hera est en effet affublé de la charge ou surcharge, ou (sur)poids encore, qu' a représenté pour elle l'épreuve de séparation d'avec son mari et les conséquences de cette séparation pour elle, en elle devrait-on plutôt dire;

- le corps souffrant et douloureux de Christiane témoigne et révèle à son tour, et à sa façon, la subsistance dans le temps des modalités de rencontre avec l'objet (objet primaire puis tenants lieu ultérieurs de celui-ci), en l'occurrence rencontres avec l'objet qui se sont déroulées sous les auspices de la douleur, physique et psychoaffective, sous les auspices encore de l'absence compensée par la présence douloureuse.

Un troisième exemple clinique va nous permettre d'enrichir et d'approfondir ces réflexions. Il s'agit d'un cas

11 Si nous osons ce néologisme. emprunté à Dejours (1989), en l'occurrence le cas de sa patiente prénommée Philippa. La cure analytique de cette patiente va être scandée à un moment donné par différents agirs de la patiente. Le premier, c'est une grave somatisation gynécologique qui conduit même la patiente à l'hôpital: Philippa a en effet présenté une rupture kystique hémorragique, ayant d'ailleurs fait craindre pour sa vie. Plus tard, ayant repris la voie du divan, la patiente est conduite à envisager une chirurgie: Philippa veut en effet se faire refaire le nez; et un beau jour elle revient de fait avec un nouveau nez. Comment comprendre pareils agirs, et leur survenue, qui plus est en regard du processus analytique? A quelles élaborations donnent-ils lieu chez la patiente - et chez son analyste? Le travail psychanalytique conduit autour de la somatisation utérine permet de révéler et de mettre à jour un épisode lointain et reculé de la vie de Philippa, un épisode qui touche à ses origines, à sa conception même, à savoir le désir infanticide de sa mère à son égard. En effet, lorsqu'elle était enceinte de Philippa, sa mère a tenté à maintes reprises d'avorter de cette enfant non désirée, voire indésirable, mais en vain donc. La suite des relations entre mère et fille n'a pas été plus satisfaisante que cette origine. Pour en revenir à Philippa adulte et à sa somatisation utérine, on ne peut manquer de faire un lien ici entre son trouble actuel et son histoire fœtale. Son trouble somatique vient en quelque sorte mettre en acte et réactualiser (par les hémorragies) cette histoire dramatique qui présida à sa venue au monde. Il est important de noter ici, dans cet exemple, que c'est la voie du corps qui a permis à Philippa de retrouver ce souvenir enfoui au fond d'elle-même et ensuite de perlaborer cette histoire. Avant d'aller plus loin, interrogeons cette idée de souvenir à propos des intentions infanticides (agies) maternelles: en effet, peut-on légitimement soutenir qu'il s'agissait réellement de souvenir chez Philippa? La psychanalyste Aulagnier (1984) s'est interrogée sur la présence de tels souvenirs datant des premières semaines suivant la naissance voire référant à la vie intra-utérine du sujet (comme c'est le cas chez Philippa donc). Aulagnier reconnaît qu'il s'agit en général chez les patients d'authentiques souvenirs mais ceux-ci sont antidatés, projetés vers cette origine au sujet de laquelle manquent des informations. Pour notre part, plutôt que de reconnaître à ces représentations pré-natales le statut de souvenirs, nous sommes davantage encline à penser qu'il s'agit de traces d'éprouvés (traumatiques certes ici) référant à l'histoire fœtale ou précoce, de telles traces permettant ultérieurement, via l'actualisation somatique, un travail psychique de représentation, d'historicisation et d'élaboration. Aulagnier précise que l'évocation de ces souvenirs s'accompagne chez le sujet d'un vécu actuel. Tel est bien en effet ce que l'on observe chez Philippa, amenée à vivre et éprouver au présent de son Moi cet épisode somatique d'une perte hémorragique non sans lien avec les tentatives abortives maternelles d'autrefois. C'est donc l'agir gynécologique qui a permis de faire surgir, chez la patiente, cet éprouvé intra-utérin, ou plutôt la trace de cette expérience précoce (ou ce traumatisme précocissisme et les représentations ultérieures afférentes). Il en est de même à propos du second agir, celui qui vise le nez: le travail analytique permet de mettre en évidence que par cet agir chirurgical (certes accompli par un autre, sur le corps de Philippa), la patiente vient alors et secondairement 
signifier qu'un nouveau-né (un nourrisson) tout neuf, et surtout bien vivant, peut maintenant advenir - peut naître de l'analyse. Le récit de ce cas entre bien en résonance avec notre propos, car le type de trouble somatique développé par la patiente (trouble gynécologique ici) est très étroitement en lien avec des expériences passées, primitives (fœtales ici), portant sur la constitution de son être psychosomatique, expériences survenues chez elle avant toute accession au langage. La somatisation génitale de Philippa actualise et reproduit même activement les fantasmes mortifères et plus encore les manœuvres abortives maternelles. Le corps se souvient-il ici..., comme le plaidait Janov? Nous sommes plutôt tentée de dire que ce trouble somatique, nullement anodin du point de vue de sa localisation anatomique, témoigne de la rémanence chez Philippa, dans son psychosoma et via l'expression de celui-ci, d'un vécu létal, vécu traumatique au sens de non réellement élaboré, non psychicisé jusqu'alors, mais qui va désormais pouvoir l'être, qui va pouvoir s'inscrire dans la topique subjective. L'inscription psychique est rendue possible de par l'actualisation de cette réalité anhistorique dans la réalité présente et surtout de par son élaboration sur la scène transféro-contre-transférentielle. Il aura néanmoins fallu à Philippa passer par ces deux agirs pour faire advenir et traiter sur la scène psychique et psychanalytique ce qui, chez elle, était en souffrance, c'est-à-dire en manque d'appropriation subjective, c'est-à-dire encore en reste ou en instance d'appropriation.

C'est ici que s'ouvre désormais un nouveau volet dans notre réflexion, à savoir que les situations ou vécus en demeure d'être traités par le sujet, qui ne peuvent l'être dans un premier temps par la voie mentale (par la voie de représentations mentales), pourraient bien l'être, selon une autre voie, la voie somatique en l'occurrence. C'est ce que nous allons maintenant développer.

\section{Le poids du passé. Ou d'hier à aujourd'hui: quand la mémoire prend corps}

En appui sur les hypothèses freudiennes, Roussillon (2003) a rappelé qu'il existait au fond trois grands types de souffrances humaines; il existerait:

- des souffrances dues à l'existence de réminiscences chez l'individu; c'est par exemple la situation prototypique de l'hystérique telle que rappelée précédemment;

- des souffrances dues à la réactualisation de ces réminiscences sous l'effet de circonstances de la réalité extérieure par exemple;

- enfin, des souffrances correspondant au retour hallucinatoire d'expériences insuffisamment appropriées subjectivement.

Le trouble somatique correspondrait, selon cette conception, à cette troisième forme de souffrances. Roussillon écrit en effet que la solution bio-logique, ou plutôt le processus de cette solution pourrait "contribuer à produire l'affection somatique (...) en infiltrant hallucinatoirement des perceptions et sensations actuelles du soma" (1999, p. 32). Pour le dire selon une autre terminologie, ce qui est forclos au dedans de la psyché va faire retour par la périphérie et par la voie du corps ${ }^{12}$.

12 Nous préférons dire par la périphérie plutôt que du dehors comme cela s'entend dire d'habitude, car une telle affirmation situerait le corps en
Ce qui est en jeu ici, c'est donc l'idée que le corps, l'expression somatique, les troubles psychosomatiques même, seraient une voie de frayage aux représentations mentales (Carels, 1987); ils seraient, pour certains individus et même potentiellement pour tout sujet selon le cours de son existence, une voie de passage possible, parfois même nécessaire, pour favoriser l'advenue de contenus psychiques et de processus psychiques d'élaboration et de symbolisation, une "voie de régulation" (Debray, 2001). Ces "passages par le corps" (selon la formulation déjà ancienne de Mathieu, 1977/1989) constitueraient donc une voie d'expression voire de symbolisation ${ }^{13}$ des conflits ou même des impensés, individuels, intersubjectifs et généalogiques (cf. un peu plus loin). C'est pourquoi en 1989 Dejours a-t-il proposé de parler de "somatisations symbolisantes" pour désigner des troubles somatiques survenant en dehors de processus de désorganisations progressives ${ }^{14}$. La caractéristique de ces somatisations symbolisantes serait de permettre "la réactivation d'un processus de création des représentations de choses quand celles-ci ont précocement été interrompues" (Dejours, 1989, p. 26). De telles somatisations, écrit-il encore, "fonctionnent pour l'appareil psychique comme précurseurs des représentations mentales" (idem). Il faut alors ajouter et reconnaître que ces situations somatiques, créatrices de liaisons psychiques, vont aussi permettre de faire advenir ce qui jusqu'alors n'avait pu être traité par la psyché, ce qui était en attente d'être subjectivé (mentalement); autrement dit ce qui vient justement de prendre corps (in-corpore) à travers ces troubles somatiques, et qui a fait le lit de la somatisation.

A propos d'impensé, et d'incorporation, nous pensons ici à certains patients obèses qui, ainsi que l'a montré Rubin (1997), logent dans leur corps un hôte, un autre, qu'ils sont chargés de porter, de nourrir - d'où le fait que ces patients là "mangent pour deux" justement. Ces patients obèses ont la charge d'entretenir et de conserver, à leur corps défendant, la mémoire de cet Autre, lequel n'est autre bien souvent qu'un objet perdu du parent dont le deuil n'a justement pu être fait par celui-ci ${ }^{15}$. D'où cet héritage psychique et physique qui incombe au descendant, ici le sujet obèse, à savoir: constituer une crypte pour maintenir cet autre en vie, perpétuer sa mémoire; et le lieu de cette crypte n'est autre que le propre psychosoma du sujet obèse. Psychosoma qui constitue alors un véritable "théâtre" (Mc Dougall, 1982, 1989) où se déroulent certains actes, parfois drames, dont Mc Dougall précise d'ailleurs qu'il s'agit de drames 'forclos de la connaissance du Je", que le travail clinique et psychanalytique, par exemple, permettra de faire apparaître en pleine lumière - à l'instar des exemples précédemment rapportés.

Ces différents éléments théorico-cliniques et cette dernière référence à l'incorporation nous ramènent plus directement à cette question des liens et rapports existant entre corps et

position d'extériorité au sujet, ce qui, personnellement, ne nous semble pas acceptable ou réaliste; le corps fait partie intégrante du sujet; à tout le moins peut-on dire que le corps occupe une position d'entre deux, entre dedans et dehors.

13 De symbolisation primaire à n'en pas douter.

14 Des désorganisations sous-tendues par les instincts de mort, comme le stipule la théorie de Marty.

15 Il peut également s'agir d'un deuil non fait du sujet lui-même. 
mémoire. Dans le contexte théorique rappelé, le trouble ou l'agir somatique n'apparaît en effet pas (ou pas systématiquement si l'on veut rester prudent) comme une entrave au travail psychique ni même comme obstacle au travail de la mémoire ou de la remémoration. Au contraire, il est ce qui le rend possible. Sans vouloir nous écarter de notre propos, il faut ici rappeler que l'agir, de manière générale, loin d'être un obstacle à la remémoration comme le pensait Freud initialement, constitue aussi, comme nombre d'auteurs le reconnaissent aujourd'hui, "une façon de se souvenir" (Potamianou, 1990).

Pour en revenir au trouble ou agir psychosomatique, on peut alors dire de lui, dans ce contexte, qu'il constitue un auxiliaire, un atout de ces divers processus et activités psychiques. Andreoli signalait déjà en 1985 comment la maladie somatique paraîtrait devoir être considérée comme un allié précieux du travail thérapeutique de la mémoire. L'auteur écrivait en effet que ce sont bien "...les objets préconscients qui paraissent soudain avoir pris forme au cours de l'éclosion psychosomatique et s'être offerts comme instruments de liaison de la pulsion" (Andreoli, 1985, p. 849). Si globalement nous souscrivons à l'idée de l'auteur, à la différence de lui toutefois, nous ne pensons pas que l'on puisse réellement parler ici de représentations préconscientes ou même de souvenirs (cf. infra); il semble plutôt s'agir de traces non représentées - non représentatives; de ce fait il s'agit de traces traumatiques. L'obésité de Mme Hera, le corps douloureux de Christiane, la somatisation utérine de Philippa constituent et véhiculent, pour chacune de ces femmes, le rappel ou plutôt la trace d'une expérience traumatique. Leur trouble est une forme d'inscription corporelle de cette trace traumatique, la trace incarnée ou engrammée dans le soma d'une expérience psycho-affective pénible, douloureuse. On peut aussi dire que le sujet s'est, avec ce trouble, cristallisé, aménagé autour de ce point de rupture qu'est l'événement ou le temps traumatique. Avec et par ce trouble est ainsi conservée la mémoire de ce passé douloureux. Car même si le trouble (somatique ici) introduit un changement, une rupture parfois radicale dans la continuité de l'existence du sujet (il y a l'avant-trouble et l'après-constitution de celui-ci); le trouble est alors véritablement - comme le dit Dupont (1972, p. 151) de la métamorphose mythologique - "un récit, un rappel de ce qui s'est passé avant la métamorphose du sujet". Vécu traumatique passé, conservé ou mémorisé dans l'expression somatique, et qui peut (re)surgir verbalement dès lors que s'offrent au sujet les conditions d'une énonciation possible. Ainsi en est-il de Mme Hera lorsqu' elle vient nous consulter: alors que nous la sollicitons sur ce qu'elle vit présentement avec/dans l'obésité, elle ouvre d'emblée la porte du passé réveillant les charges traumatiques restées jusqu'alors en latence chez elle. Il en est de même avec Christiane, dans une temporalité un peu plus longue que celle de Mme Hera, et de même encore dans le cas de Philippa qui pourra seulement après cette grave expérience maladive identifier et travailler la haine maternelle à son endroit.

Par l'expression somatique - le trouble en soi (la chose) et ce que celui-ci suscite, les mots - le sujet (re)trouve donc la trace des expériences subjectives, dont les charges conflictuelles, et plus encore surexcitantes et traumatiques, avaient été occultées pour un temps. Lors de la démarche de consultation ${ }^{16}$, l'occulté - ou l'occulte, c'est-à-dire ce qui existait, subsistait dans les limbes (autrement dit, dans un état vague et incertain) - soit encore le forclos fait retour. Celuici ne s'effectue pas seulement par la voie mentale - comme dans le cas de l'hallucination psychotique et du délire - mais par la voie somatique, au moyen de la chose corporelle - la surcharge physique chez Mme Hera, la douleur physique chez Christiane, la pathologie utérine chez Phillipa. Celleci fournit au sujet l'occasion de trouver et de dire les mots d'une souffrance jusqu'alors impensée, non reconnue, non subjectivée à certains égards, de par l'impact du traumatisme. Par la voie du soma donc, mais pas selon la manière dont s'effectue le retour du refoulé névrotique hystérique, lequel témoigne, lui, de contenus mentalisés, secondarisés, de contenus psychiques inconscients (cf. "inconscient sexuel" de la troisième topique de Dejours, 2001). Même si les différents troubles présentés par les trois patientes évoquées sont bien différents ${ }^{17}$, ils correspondent chacun à une sorte de mise en latence ou mise en suspens de certains éléments, de charges et vécus passés et traumatiques non historicisés. Ceux-ci n'ont nullement disparu, ne se sont nullement éteints; ils couvent sous le trouble, quelque soit la nature de celui-ci, et ils peuvent resurgir à la faveur du trouble lui-même, à partir de celui-ci, constituant simultanément un point d'ancrage, un point d'étayage et de frayage à la représentation.

Le trouble somatique (ou dit psychosomatique) montre alors comment chez certains individus le soma s'est en quelque sorte mis au diapason avec la réalité psychique et subjective, ou plutôt avec la réalité psychique et subjective non reconnue mais en demeure ou voie d'advenir, un jour peut-être. Réceptacle potentiel du non mentalisé ou non métabolisé, le corps apparaît ainsi comme un lieu d'inscription possible de ce qui, chez le sujet, a momentanément échoué à s'inscrire sur la scène psychique. A ce titre le corps vient tenir lieu de topique (topique extra-psychique ou parapsychique), topique au sens d'un conteneur possible des éléments en attente et en instance de figuration autre (autre que somatique), en instance d'intégration et d'historicisation. Le trouble psychosomatique apparait bien alors doté d'une fonction de mémoire: inscription, mémorisation et/ou rappel d'un trouble, d'une crise, d'un trauma (Delourmel, 2002) ou d'une expérience non subjectivée.

Ceci rejoint ou plutôt correspond à la troisième forme de mémoire décrite par Roussillon (2003). Selon lui, il existerait en effet trois formes de mémoire: une première, celle du souvenir (qui correspond à une représentation vécue comme telle et datée, représentée comme passée; c'est une mémoire qui suppose donc un travail de représentation et de datation); une seconde mémoire qui est celle du fantasme (et laquelle se donne comme hors temps); et enfin, une troisième mémoire, celle qui nous intéresse surtout ici, qui est une "mémoire paradoxale" dans la mesure où, dit l' auteur, "elle ne se donne ni comme souvenir ni comme représentation psychique mais comme perception, comme sensation, voire comme affect

16 Démarche révélatrice à cet égard d'une certaine prise de conscience du sujet relativement à son état ou équilibre psychosomatique.

17 Rien de comparable en effet, manifestement, entre l'obésité de Mme Hera qui se constitue progressivement (bien que rapidement) en au fil du temps et la brutale désorganisation utérine qui éclôt soudainement chez Philippa. 
actuel; elle re-présente sans le savoir un pan de l'histoire vécue non subjectivé ni historisé, se donnant comme actuel et agissant" (Roussillon, 2003, p. 141). Soit, ce que pour notre part, nous avons tenté de rendre compte sous le terme de rémanence, et dont le psychosoma du sujet obèse (Mme Hera) ou d'autres patients porteurs de troubles somatiques (Christiane, Philippa) nous donne à percevoir et penser.

\section{Conclusion}

Pour conclure, nous reprendrons les propos d' Andreoli concernant les éclosions psychosomatiques. Il écrivait en effet que "ces manifestation corporelles jouent un rôle majeur dans la mobilisation de la trace des investissements fondamentaux de l'histoire du patient' (1985, p. 836). Le trouble (psycho)somatique apparaît dès lors bien constituer une forme, obscure, de mémoire ou du moins une condition qui met en jeu les rapports du sujet à sa mémoire, quoique sous des formes bien différentes de celles de l'hystérie de conversion (auxquelles certains auteurs on eu le tort de réduire l'économie de l'éclosion psychosomatique). C'est ici que nous rejoignons alors l'assertion d'Adomnicai (1991, p. 371) selon laquelle le malade somatique ne souffre pas de réminiscences comme l'hystérique, le malade somatique, lui, "souffrirait pour avoir des réminiscences". Maladie somatique et trouble de conversion entretiennent bien tous deux un rapport à la mémoire (Andreoli, 1985), mais sous des formes différentes ou bien différenciées qu'il convient de garder nettement à l'esprit. Tandis que le corps de l'hystérique est le siège de représentations, convoque et charrie des matériaux refoulés, le corps du patient somatique, lui, stocke, engramme et tient en réserve (et tient donc à disposition, à demeure) des expériences d'un autre ordre, traumatiques au sens de non historicisées, des expériences siégeant sans doute alors du côté de "l'inconscient amential" (Dejours, 2001), des expériences infra-verbales peut-être aussi et de nature plus archaïque $^{18}$ mais qui restent à tout moment susceptibles d'être réveillées, mobilisées, pensées, parlées. Les histoires de Mme Hera, Christiane et Philippa nous ont fourni chacune, et chacune selon un mode somatique singulier, une vivante illustration du corps porteur de traces pesantes et traumatiques du passé.

\section{References}

Adomnicai, I. (1991). Compulsion de répétition et maladie somatique. Adolescence, 9(2), 369-384.

Andreoli, A. (1985). Corps et mémoire en psychothérapie et en psychanalyse. L'Évolution Psychiatrique, 50(4), 835-854.

Anzieu (Org.)(1977). Psychanalyse et langage. Paris: Dunod, 1989.

Aulagnier, P. (1984). L'apprenti historien et le Maître sorcier. Du discours identifiant au discours délirant, Paris: PUF.
Carels, N. (1987). Les liens entre corps, agir et préconscient. Revue Française de Psychanalyse, 51(2), 665-680.

Célérier, M-C. (1989). Corps et fantasme. Pathologie du psychosomatique, Paris: Dunod.

Debray, R. (2001). Epître à ceux qui somatisent. Paris: PUF.

Dejours, C. (1989). Recherches psychanalytiques sur le corps. Répression et subversion en psychosomatique. Paris: Payot.

Dejours, C. (2001). Le corps d'abord, Paris: Payot.

Delourmel, C. (2002). Traumatisme et mémoire: événement et/ou trace?. Revue Française de Psychanalyse, 3, 711-727.

Del Volgo, M-J. (1993). L'instant de dire. Cliniques Méditerranéennes, 37/38, 63-77.

Dumet, N. (2004). Le corps du thérapeute, lieu d'actualisation transféro-contre-transférentielle. Filigrane, 13(2), 81-95.

Dupont, F. (1972). Se reproduire ou se métamorphoser. Topique, 9/10, 139-160.

Freud, S. (1953). La dynamique du transfert. Em S. Freud (Org.), La technique psychanalytique (pp. 50-60). Paris: PUF. (Trabalho original publicado em 1912)

Janov, A. (1997). Le corps se souvient. Paris: Editions du Rocher.

Kandel, E. (2002). La biologie et le futur de la psychanalyse: un nouveau cadre conceptuel de travail pour une psychiatrie revisitée. L'Évolution Psychiatrique, 67, 40-82.

Larousse (1993). Dictionnaire de la langue française, lexis. Paris: Larousse.

L'heureux-Le-beuf, D. (2002). Trajectoires hypocondrie-soma. Revue Française de Psychosomatique, 22, 9-20.

Marty, P. (1976) Les mouvements individuels de vie et de mort. Tome 1. Essai d'économie psychosomatique, Paris: Payot.

Marty, P. (1980). L'ordre psychosomatique. Tome 2. Désorganisations et régressions. Paris: Payot.

Marty, P. (1990). La psychosomatique de l'adulte. Paris: PUF.

Mathieu, M. (1989). Dont acte. Em D. Anzieu (Org.), Psychanalyse et langage (pp. 139-162). Paris: Dunod. (Trabalho original publicado em 1977).

Mc Dougall, J. (1982). Theâtres du Je. Paris: Gallimard.

Mc Dougall, J. (1989). Theâtres du corps. Paris: Gallimard.

Potamianou, A. (1990). Rêves et somatisation. Topique, 45, 49-62.

Roussillon, R. (1999). Agonie, clivage et symbolisation. Paris: PUF.

Roussillon, R. (2003). Historicité et mémoire subjective. La troisième trace. Cliniques Méditerranéennes, 67, 127-144.

Rubin, G. (1997). Cannibalisme psychique et obésité. Paris: Delachaux et Niestlé.

Stoloff, J-C. (2000). Interpréter le narcissisme. Paris: Dunod.

Varela, F. (1993). L'inscription corporelle de l'esprit. Paris: Seuil.

Recebido em 06.10.2007

Primeira decisão editorial em 06.11.2007

Versão final em 05.01.2008

Aceito em 10.02.2008

18 Comme Mc Dougall (1989) par exemple l'a théorisé de son côté sous le vocable d'hystérie archaïque. 